\title{
Indicadores ambientales y gestión urbana. Relaciones entre servicios ecosistémicos urbanos y sustentabilidad
}

\author{
Clara María Karis \\ Arquitecta y magíster en Gestión Ambiental del Desarrollo \\ Urbano (UNMdP). Doctoranda en Arquitectura y Urbanismo \\ en la Universidad Nacional de Mar del Plata, con el financia- \\ miento de una beca interna doctoral del CONICET. Instituto \\ del Hábitat y del Ambiente de la Universidad Nacional de Mar \\ del Plata. \\ clarakaris@hotmail.com \\ Camila Magalí Mujica \\ Lic. en Diagnóstico y Gestión Ambiental por la Universidad \\ Nacional del Centro. Doctoranda en Ciencias Aplicadas, Men- \\ ción Ambiente y Salud en la misma Universidad, con el finan- \\ ciamiento de una beca doctoral del CONICET en temas estra- \\ tégicos. Instituto del Hábitat y del Ambiente de la Universidad \\ Nacional de Mar del Plata. \\ camilamagalimujica@gmail.com

\section{Rosana Ferraro} \\ Lic. en Ecología y Conservación de los Recursos Naturales Re- \\ novables (UNLP) y magíster Sciencie en Gestión Ambiental del \\ Desarrollo Urbano (UNMdP). Docente-investigadora en el Ins- \\ tituto del Hábitat y del Ambiente de la FAUD (UNMdP). \\ rosanaferraro_2@hotmail.com
}

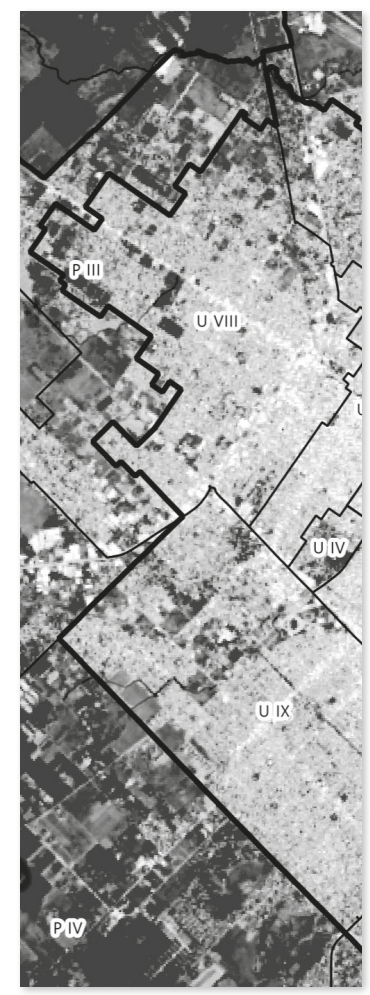




\section{Indicadores ambientales y gestión urbana. Relaciones entre servicios ecosistémicos urbanos y sustentabilidad}

\section{Resumen}

Las ciudades enfrentan desafíos de sustentabilidad que hacen necesario generar información ambiental a través del uso de indicadores. El presente trabajo analiza la disponibilidad y distribución de infraestructura ecológica en la ciudad de Mar del Plata y su periurbano (Argentina), de manera tal de construir una base de información necesaria para evaluar la disponibilidad de Servicios Ecosistémicos Urbanos. Para ello, se utilizaron indicadores ambientales que evalúan dichos atributos en la superficie verde pública, la superficie de suelo no impermeabilizado y la cubierta de vegetación. Los resultados muestran una desigual distribución de dichos elementos, lo cual podría tener implicancias en las oportunidades de contacto directo con la naturaleza por parte de la población urbana, siendo esto un determinante en la generación de gran parte de los Servicios Ecosistémicos Culturales.

\section{Palabras clave}

Espacios verdes; infraestructura ecológica; servicios ecosistémicos culturales; sustentabilidad urbana.

\section{Environmental indicators and urban management. Relations between urban ecosystemic services and sustainability}

\section{Abstract}

Cities face sustainability challenges that make it necessary to generate environmental information through the use of indicators. This paper analyzes the availability and distribution of ecological infrastructure in the city of Mar del Plata (Argentina), and its peri-urban area, in order to build a necessary information base to assess the availability of Urban Ecosystemic Services.

For this, environmental indicators were used that evaluate these attributes on the public green surface, the surface of non-waterproofed soil and the vegetation cover. The results show an unequal distribution of these elements, which could have implications for opportunities for direct contact with nature by the urban population, this being a determinant in the generation of a large part of the Cultural Ecosystemic Services.

\section{Keywords}

Green areas; ecological infrastructure; cultural ecosystemic services; urban sustainability.

\section{Indicadores ambientais e gestão urbana. Relações entre serviços ecossistêmicos urbanos e sustentabilidade}

\section{Resumo}

As cidades enfrentam desafios de sustentabilidade que tornam necessário gerar informações ambientais por meio do uso de indicadores. Este artigo analisa a disponibilidade e distribuição de infraestrutura ecológica na cidade de Mar del Plata (Argentina), e sua área periurbana a fim de construir uma base de informações necessária para avaliar a disponibilidade dos Serviços Ecossistêmicos Urbanos. Para isso, foram utilizados indicadores ambientais que avaliam esses atributos na superfície verde pública, na superfície do solo não impermeabilizado e na cobertura vegetal. Os resultados mostram uma distribuição desigual desses elementos, o que pode ter implicações em oportunidades de contato direto com a natureza pela população urbana, sendo este um fator determinante na geração de grande parte dos Serviços Ecossistêmicos Culturais.

\section{Palavras chave}

Espaços verdes; infraestrutura ecológica; serviços ecossistêmicos culturais; sustentabilidade urbana. 


\section{Introducción}

La infraestructura ecológica, también llamada infraestructura verde (BENEDict y MсмAнon, 2002; European Environment Agency, 2011; Gómez-Baggethun et AL., 2013), comprende todas las redes naturales, seminaturales y artificiales de los sistemas ecológicos presentes en el interior y la periferia de las zonas urbanas (Tzoulas ET AL., 2007). El hecho de considerar dichos elementos como un tipo de infraestructura, de forma análoga a la infraestructura construida, surgió en las últimas décadas para cambiar la percepción acerca de estos espacios y darles mayor entidad dentro de los procesos de planificación, lo cual involucra, inevitablemente, la necesidad de ser estratégicamente diseñados y mantenidos (SANDSTRöM, 2002).

A su vez, este concepto hace referencia al rol de dicha infraestructura en la provisión de Servicios Ecosistémicos Urbanos (SEU) en beneficio de los habitantes (Breuste, HaAsey ELmQvist, 2013; Civeira, Lado Liñares, Vidal Vázquez y Paz GonzÁlez, 2018; Gómez-Baggethun y Barton, 2013; Keeler et AL., 2019). Desde una perspectiva socio-ecológica (Grove, 2009; MarTín-López, Gómez-Baggethun y Montes, 2009), los SEU pueden ser definidos como los beneficios que las personas obtienen directa o indirectamente de los ecosistemas urbanos y sus componentes (Gómez-BAGgethun et AL., 2013; MEA, 2003). Estos generalmente se clasifican en tres grandes grupos (Haines-Young y Potschin, 2018): 1) servicios de suministro o aprovisionamiento: productos que se obtienen de los ecosistemas, como los alimentos y el agua; 2) servicios de regulación: beneficios que se obtienen de la regulación de los procesos de los ecosistemas, como la regulación de las inundaciones y el clima y 3) servicios culturales: beneficios intangibles que las personas obtienen de los ecosistemas. Estos comprenden tanto los beneficios que se obtienen de la interacción directa con la naturaleza, cuya generación depende de la presencia de las personas en el sitio, como aquellos que se obtienen de forma indirecta, tales como los de índole espiritual o simbólica.

El presente trabajo se enfoca en los SEU culturales, haciendo especial hincapié en aquellos que se obtienen de la interacción directa con la naturaleza (entre los que se destacan las oportunidades para la realización de actividades deportivas y de ocio, la contemplación de la naturaleza y la educación y la investigación). Este tipo de SEU reviste un interés particular en el interior de las ciudades, dado que los espacios verdes que forman parte de la infraestructura ecológica constituyen, en muchos casos, los únicos sitios donde los habitantes urbanos pueden tener contacto directo con la naturaleza. 
La producción de estos servicios depende tanto de factores ecológicos como de factores sociales, dado que son el resultado del procesamiento de información del entorno biofísico a través de los sentidos humanos (BRAAT y DE GRoot, 2012). No obstante, estudios antecedentes indican que su generación se ve condicionada por las características de la infraestructura ecológica, como por ejemplo, la superficie, la distribución de los elementos que la componen y la proximidad que tienen con los habitantes (Karis y Ferraro, 2017; Reyes Päcke y Figueroa Aldunce, 2010).

En relación con la superficie, se advierte que los espacios de gran tamaño permiten el desarrollo de mayor diversidad y riqueza de especies vegetales y de fauna (BReuste, SCHNELLINGER, QuRESHIY FAGGI, 2013), lo que se relaciona con las posibilidades de contemplar la naturaleza. A su vez, el mayor tamaño de los espacios verdes públicos promueve la realización de diversas actividades de recreación y ocio, y facilita la presencia simultánea de distintos grupos, como niños, adultos y jóvenes (Reyes Päckey Figueroa Aldunce, 2010).

Asimismo, se ha observado una estrecha relación entre los SEU culturales y el lugar donde son producidos (Dickinson y HobBs, 2017). Estos servicios no pueden reproducirse exactamente con las mismas características en distintos lugares y, como se mencionó previamente, muchos dependen de la presencia de las personas en el sitio. Por lo tanto, la distancia podría afectar las posibilidades que poseen los habitantes urbanos de disfrutar con regularidad de este tipo de servicios, siendo deseable que todos los barrios de las ciudades cuenten con espacios vegetados. En este sentido, existe una relación directa entre el tamaño de estos espacios y su área de influencia: mientras que los de mayor tamaño pueden ser menos en cantidad y estar más alejados entre sí, los más pequeños debieran encontrarse a una distancia tal que se pueda acceder cotidianamente a pie (Coles y Bussey, 2000; Reyes Päcke y FigueroA AlduncE, 2010).

En los entornos urbanos, donde el paisaje natural ha sido intensamente modificado, las características mencionadas son objeto de planificación y diseño, por lo que la generación de SEU culturales se ve influenciada por las prácticas y políticas de gestión urbana (ANDERSson, BARTHEL y AHRNÉ, 2007). A menudo, en las grandes ciudades latinoamericanas, los espacios verdes y azules son escasos como consecuencia del acelerado y precario proceso de urbanización ocurrido en la segunda mitad del siglo XX (Reyes Päcke y FigueroA Aldunce, 2010), que promovió especialmente el crecimiento de ciudades intermedias con poblaciones de 
entre 100.000 y 2.000 .000 de habitantes (Banco Interamericano de Desarrollo, 2016). En estas ciudades, como es el caso de Mar del Plata, los procesos de crecimiento urbano muchas veces son de tipo "espontáneo" (Zulaica y FerRaro, 2013), y, por lo tanto, sin una planificación basada en la infraestructura ecológica ni con criterios tendientes a promover o potenciar los SEU.

Dados los desafíos de sustentabilidad que deben enfrentar las ciudades, resulta necesario generar información ambiental que permita monitorear los fenómenos urbanos sobre la base de un conjunto de evidencias. Entre estos se encuentran los modelos de indicadores, los cuales constituyen herramientas de objetivación de procesos ambientales que transforman información en conocimiento social (Quiroga MarTínez, 2009). En el ámbito de la gestión urbana, los indicadores son una herramienta de información valiosa para conocer el estado de un municipio, en tanto permiten generar evidencias en los procesos de decisión y monitoreo de un determinado proceso y evaluar su progreso, ya sea en relación con determinadas metas cuando estas existen, o con respecto a los niveles observados en un año base (Agencia de Ecología Urbana de Barcelona, 2007; Quiroga Martínez, 2009).

Para evaluar la situación de una ciudad en relación con su infraestructura ecológica y sus SEU, podrían utilizarse indicadores ambientales, dado que dependen de procesos que pueden ser cuantificados sobre la base de las estructuras que los proveen o de sus funciones (De La BARRera, Reyes-PAeckey Banzhaf, 2016). En este sentido, para generar información sobre los SEU culturales, podrían cuantificarse los espacios verdes públicos, de gran importancia por su accesibilidad, y también otros tipos de áreas verdes, como las situadas en áreas residenciales, que en algunos casos han demostrado ser las principales proveedoras de algunos servicios por su mayor superficie (Breuste et al., 2013; Loram, Tratalos, Warren y Gaston, 2007).

En Mar del Plata actualmente no existe suficiente información sistematizada sobre los SEU culturales ni sobre la infraestructura ecológica que los provee. El único dato específico con el que cuenta la ciudad surgió del diagnóstico realizado por la Iniciativa Ciudades Emergentes y Sostenibles (ICES) del Banco Interamericano de Desarrollo (BID) ${ }^{1}$, de la cual Mar del Plata forma parte. Dicho diagnóstico evaluó, entre otros aspectos de la sustentabilidad urbana, la disponibilidad de áreas verdes dentro del ejido urbano de la ciudad a partir de las áreas verdes por cada 100.000 habitantes. Sin embargo, este indicador no ofrece información acerca de la distribución espacial de dichos espacios dentro del área de
1. ICES es un programa de asistencia técnica no reembolsable del BID que emplea un enfoque integral e interdisciplinario para identificar, organizar y priorizar intervenciones urbanas para hacer frente a los principales obstáculos que impiden el crecimiento sostenible de las ciudades emergentes de América Latina y el Caribe. Este enfoque transversal se basa en tres pilares: (1) sostenibilidad medioambiental y de cambio climático; (2) sostenibilidad urbana y (3) sostenibilidad fiscal y gobernabilidad. 
2. En relación con este indicador, ha sido ampliamente citada una recomendación de la Organización Mundial de la Salud (OMS) de acuerdo con la cual las ciudades deberían tener un mínimo de 9 o 10 $m^{2}$ de superficie verde por habitante, siendo deseable una dotación de $15 \mathrm{~m}^{2}$ por habitante. Sin embargo, no existe ningún documento oficial de la OMS que lo respalde. estudio. Lo mismo sucede con la versión de este indicador que expresa el resultado en $\mathrm{m}^{2} /$ hab., a pesar de ser el más ampliamente utilizado por los organismos de gestión urbana $^{2}$ (por ejemplo, Agencia de Ecología Urbana de Barcelona, 2010; Ministerio de Desarrollo Urbano Buenos Aires, 2009).

Trabajos antecedentes (KARIS, 2017) muestran que en general se observa una falta de superficie verde pública en la mayor parte de los barrios de la ciudad de Mar del Plata, y que esta se concentra en el centro de la ciudad y sobre la costa marítima. Este trabajo se plantea como hipótesis central que los espacios verdes configuran zonas que difieren cuali y cuantitativamente en su capacidad para brindar servicios ecosistémicos culturales en el interior del área urbana y periurbana, en términos de los indicadores seleccionados. En este marco, propone como objetivo general profundizar en el análisis de la disponibilidad y distribución de los elementos que componen la infraestructura ecológica de la ciudad y su periurbano, de manera tal que posibilite generar una base de información necesaria para evaluar la disponibilidad de SEU culturales. Los objetivos específicos son:

- comparar la dotación y superficie de los espacios verdes públicos, la superficie no impermeabilizada y la cubierta de vegetación en diferentes zonas del área de estudio;

- establecer una línea de base para una serie de indicadores ambientales de infraestructura ecológica.

\section{Metodología}

\section{Área de estudio y definición de zonas de análisis}

El área de estudio corresponde a la zona urbana y periurbana de la ciudad de Mar del Plata (ver figura 1). Los límites se tomaron siguiendo los criterios utilizados por ZulaICA y FeRRARO (2013), de modo tal que el espacio urbano queda definido por la presencia de amanzanamiento, agua de red y red cloacal. El periurbano queda delimitado, por un lado, por el borde rígido de lo urbano y, por otro, por uno más difuso que lo separa del territorio netamente rural, caracterizado por la presencia de agricultura y ganadería extensivas. A los límites del periurbano tomados de Zulaica y FerRaro (2013) se agregaron los barrios ubicados sobre la ruta 11 en dirección a Miramar, dado que en la actualidad constituye uno de los ejes de expansión de la ciudad (ver Zona PVI en figura 1). 
Indicadores ambientales y gestión urbana.

Relaciones entre servicios ecosistémicos urbanos y sustentabilidad



Figura 1. Área de estudio y zonas de análisis. Fuente: elaboración propia 
Para alcanzar los objetivos propuestos, en primer lugar se delimitaron zonas de análisis dentro del área de estudio. Estas se definieron utilizando como base la delimitación de barrios empleada por el Municipio de General Pueyrredón (Municipalidad de General Pueyrredón, 2017в).

En el área urbana, las zonas surgen de agrupar aquellos barrios que puedan ser considerados relativamente homogéneos en cuanto a trazado, tejido y calidad de vida. Para ello, el trazado y el tejido fueron analizados sobre la imagen satelital provista por Google Earth, mientras que los datos acerca de calidad de vida se tomaron del indicador de calidad de vida elaborado por LuCERo ET AL. (2011).

Para la definición de zonas en el periurbano, también se utilizó como base la delimitación de barrios del Municipio de General Pueyrredón (Municipalidad de General Pueyrredón, 2017в), y se agruparon aquellos considerados relativamente homogéneos en cuanto a calidad de vida. En este caso, la homogeneidad es menor, dado que las zonas de la interfase urbano-rural conforman un paisaje productivo y social altamente heterogéneo, donde se desarrollan funciones económicas, como la producción y el abastecimiento de materias primas y elaboradas, y funciones sociales, que hacen que este espacio sea concebido como lugar de residencia y de trabajo para sectores socialmente muy diferenciados (GARAY, 1999).

En ambos casos, los límites de las zonas se ajustaron a los límites de los radios censales con el objetivo de utilizar datos poblacionales provistos por el Censo Nacional de Población, Hogares y Viviendas en el desarrollo de la investigación. Quedaron así definidas diez zonas dentro del área urbana y nueve en el periurbano (ver figura 1).

\section{Indicadores ambientales}

Para analizar la disponibilidad y distribución de los elementos que componen la infraestructura ecológica en el área de estudio, se aplicó un conjunto de indicadores ambientales que evalúan la dotación de espacios verdes públicos, la superficie de suelo no impermeabilizado y la cubierta de vegetación en las zonas de análisis delimitadas previamente (ver tabla 1). Para la selección de los indicadores ambientales, se tomó como base la serie realizada por Karis y FerRARo (2017), que fue modificada de acuerdo con los objetivos del presente trabajo y la información disponible para su cálculo. 


\begin{tabular}{l|l|l}
\hline \multicolumn{2}{c}{ Conjunto de indicadores ambientales utilizados } \\
\hline \multicolumn{1}{|c}{ INDICADOR } & \multicolumn{1}{c}{ DESCRIPCIÓN } \\
\hline 1 & $\begin{array}{l}\text { Superficie verde pública por } \\
\text { habitante }\end{array}$ & $\begin{array}{l}\text { El indicador mide la extensión de las áreas } \\
\text { verdes públicas existentes y la relación con el } \\
\text { número de habitantes. }\end{array}$ \\
\hline 2 & $\begin{array}{l}\text { Distribución de la superficie } \\
\text { verde pública }\end{array}$ & $\begin{array}{l}\text { El indicador mide la participación de cada zona } \\
\text { en la superficie total de áreas verdes públicas del } \\
\text { área de estudio. }\end{array}$ \\
\hline 3 & Superficie no impermeabilizada & $\begin{array}{l}\text { El indicador mide el porcentaje de superficie de } \\
\text { suelo no impermeabilizado. }\end{array}$ \\
\hline 4 & $\begin{array}{l}\text { Índice de vegetación diferencial } \\
\text { normalizada (NDVI) }\end{array}$ & $\begin{array}{l}\text { Se utiliza el NDVI para evaluar la superficie de } \\
\text { suelo cubierta de vegetación en espacios públicos } \\
\text { y privados. }\end{array}$ \\
\hline
\end{tabular}

Fuente: elaboración propia

El indicador 1 (superficie verde pública por habitante) mide la extensión de los espacios verdes públicos existentes y la relación con el número de habitantes, expresando el resultado en $\mathrm{m}^{2} / \mathrm{hab}$. Al ser aplicado en zonas de la ciudad, permite comparar los resultados y detectar situaciones de escasez y/o desigualdad.

El indicador 2 (distribución de la superficie verde pública) mide la participación de cada zona de la ciudad en la superficie total de las áreas verdes públicas, calculada como el porcentaje de superficie verde pública que pertenece a cada zona. Al igual que el indicador 1 , tiene un valor comparativo entre zonas y permite reconocer en cuáles se concentra una mayor cantidad de superficie verde pública.

Para el cálculo de ambos indicadores se utilizaron principalmente dos insumos: los datos provistos por el Censo Nacional de Población, Hogares y Viviendas 2010 y una base de datos georreferenciada, realizada en QGis 2.16.3 y que cuenta con capas vectoriales de los 
componentes de la infraestructura ecológica en el área de estudio. Esta se elaboró sobre la base de información provista por el Instituto del Hábitat y el Ambiente (IHAM), el Código de Ordenamiento territorial del Partido de General Pueyrredón (Municipalidad de General PueYrRedón, 2017A) y la consulta de imágenes satelitales de Google Earth para el año 2017, e incluye las siguientes categorías de espacios verdes públicos:

- Plazas barriales: espacios abiertos de superficie de entre $1000 \mathrm{~m}^{2}$ y 3,5 ha donde predominan la cobertura vegetal y los elementos naturales, de acceso libre, cuyas funciones principales son la recreación, el contacto con la naturaleza y la interacción social.

- Plazas y parques urbanos: espacios abiertos de superficie de entre 3,5 y 10 ha donde predominan la cobertura vegetal y los elementos naturales, de acceso libre, cuyas funciones principales son la recreación, el contacto con la naturaleza y la interacción social.

- Parques grandes: espacios abiertos de superficie mayor a 10 ha donde predominan la cobertura vegetal y los elementos naturales, de acceso libre, cuyas funciones principales son la recreación, el contacto con la naturaleza y la interacción social.

- Áreas de reserva natural: espacios naturales que por su fragilidad, importancia o singularidad son objeto de protección legal para garantizar su conservación. Se consideran para el cálculo de los indicadores aquellos de propiedad pública, excluyéndose los barrios residenciales declarados reserva forestal.

Para el cálculo del indicador 3 (superficie no impermeabilizada), se trabajó con imágenes satelitales obtenidas de Google Earth Pro para el año 2017. Se analizó la superficie no impermeabilizada a partir de la identificación y agrupación de áreas urbanas con rasgos homogéneos en cuanto al tipo de tejido y a la relación entre la superficie construida y la libre de edificación. De cada área homogénea, se tomó una muestra de cuatro manzanas, en la que, a partir de dibujar y medir la superficie no impermeabilizada sobre las fotografías, se calculó su porcentaje, y luego se ajustaron los resultados a cuatro rangos:

- 0-25 \%: áreas de superficie que presentan mayormente suelo construido, con una presencia escasa de suelos no impermeabilizados;

- 26-50 \%: áreas con una mayor proporción de suelo con presencia de construcciones que de suelo no impermeabilizado;

- 51-75 \%: áreas con edificaciones, pero con una mayor proporción de suelos no impermeabilizados;

- 76-100 \%: áreas que presentan edificaciones dispersas con amplias zonas libres o espacios vacantes sin impermeabilizar. 
Finalmente, el indicador 4 corresponde al Índice de Vegetación Diferencial Normalizada (NDVI, por sus siglas en inglés). Este es un parámetro calculado a partir de los valores de la reflectividad a distintas longitudes de onda y que pretenden extraer de estos la información relacionada con la vegetación. Para ello, utilizan los valores de reflectividad en las zonas espectrales del rojo (R) e infrarrojo cercano (IRC). El NDVI es uno de los índices de vegetación más utilizado, debido a que posee una gran sencillez de cálculo y una fácil interpretación directa de los parámetros biofísicos de la vegetación (Gilabert, González-Piqueras y García-Haro, 1997; SAnchez Rodriguez et AL., 2000). Para su cálculo se utilizó una imagen satelital Landsat 8 en sus bandas 4, 5 y 10 (del infrarrojo térmico) obtenidas del Landsat 8 (Path/Row 224-86) del día 22/02/2018 a las 13: 39. Para obtener el índice, la imagen se procesó con el software ENVI 4.5. Se convirtieron los valores expresados en Números Digitales (ND) a valores radiométricos y luego se corrigieron las bandas en el espectro solar y en el espectro térmico. A partir de las bandas 4 y 5 se obtuvo el Índice de Vegetación Diferenciada Normalizada (NDVI), siguiendo la fórmula:

$$
\mathrm{NDVI}=\frac{\mathrm{IRC}-\mathrm{R}}{\mathrm{IRC}+\mathrm{R}}
$$

Donde IRC es la reflectividad en el infrarrojo cercano (banda 5 del Landsat 8) y R es la reflectividad en el rojo (banda 4 del Landsat 8).

El NDVI adopta valores entre -1 a 1 y puede ser interpretado de la siguiente forma:

- Valores entre - 1 y 0 corresponden a agua y nubes.

- Valores cercanos a cero corresponden principalmente a afloramientos rocosos, construcciones y terrenos desnudos o sin vegetación.

- Valores moderados (aproximadamente de 0,4 a 0,7) representan terrenos con arbustos y prados.

- Valores altos (> a 0,7) indican vegetación frondosa y en buen estado, asociada principalmente a forestaciones.

Ambos indicadores muestran la distribución del suelo no construido y cubierto de vegetación. El mapeo de estos indicadores permite localizar los sectores del área de estudio que cuentan con mayor presencia de vegetación, independientemente de su uso, así como ubicar las vías con mayor presencia de arbolado. 


\section{Resultados y discusión}

Al analizar la superficie total de espacios verdes públicos y la relación con el número de habitantes (indicador 1), se observan diferencias entre las zonas de análisis definidas. Si se comparan estos resultados con los parámetros utilizados en las iniciativas citadas (AGENCIA de Ecología Urbana de Barcelona, 2010; Ministerio de Desarrollo Urbano Buenos Aires, 2009), se advierte que del total de las zonas del área de estudio, solo 5 superan los $15 \mathrm{~m}^{2} / \mathrm{hab}$, de las cuales cuatro de ubican en el periurbano, y 13 exhiben valores por debajo de $\operatorname{los} 9 \mathrm{~m}^{2} / \mathrm{hab}$. (ver figura 2). Esto se debe a la presencia de espacios verdes de gran tamaño en algunas de las cinco zonas mencionadas, pero también al sesgo positivo que tiene este indicador en zonas con baja densidad de población (DE LA BARRERA ET AL., 2016).

El indicador 2 (distribución) refuerza los resultados obtenidos en el anterior. Este muestra una desigual distribución de la superficie verde pública en el área de estudio, con una alta concentración de aquella en la zona P1 (55,88 \%), seguida por la PVIII (11,76 \%), PVI (7,56 \%) y UX (6,40 \%) (ver figura 3). La concentración de superficie verde pública en dichas zonas se debe principalmente a la presencia de parques de grandes dimensiones y áreas de reserva natural, lo que permite una mayor diversificación de usos y SEU culturales. Sin embargo, los valores obtenidos en las zonas PIV (0,04 \%), UIV (0,07\%) y UII (0,30 \%) evidencian la escasez de espacios verdes públicos en las cuatro categorías identificadas. En este sentido, sería deseable la existencia de una mayor cantidad de superficie verde dispersa en parques de menor tamaño, de manera tal de garantizar el contacto regular de los habitantes urbanos con la naturaleza.

Si bien los espacios verdes públicos son de gran importancia debido a su accesibilidad y funciones de recreación e interacción social entre los habitantes, algunos estudios demostraron que en determinadas ciudades las áreas residenciales son cuantitativamente las mayores proveedoras de beneficios por su mayor superficie (BREuste ET AL., 2013; LoRAM et AL., 2007). A pesar de no brindar oportunidades para la interacción social, sí ofrecen la posibilidad de contacto con la naturaleza para los habitantes de estas zonas, dado que cobran importancia sus cualidades estéticas.

Estos aspectos fueron evaluados sobre la base de los indicadores 3 y 4, cuyos resultados permiten afirmar que, dentro del área urbana, el centro de la ciudad y las zonas adyacentes son las que presentan tejido más denso. En estas, la superficie de suelo sin impermeabilizar 
Indicadores ambientales y gestión urbana.

Relaciones entre servicios ecosistémicos urbanos y sustentabilidad



Figura 2. Indicador 1. Superficie verde pública por habitante. Fuente: elaboración propia 


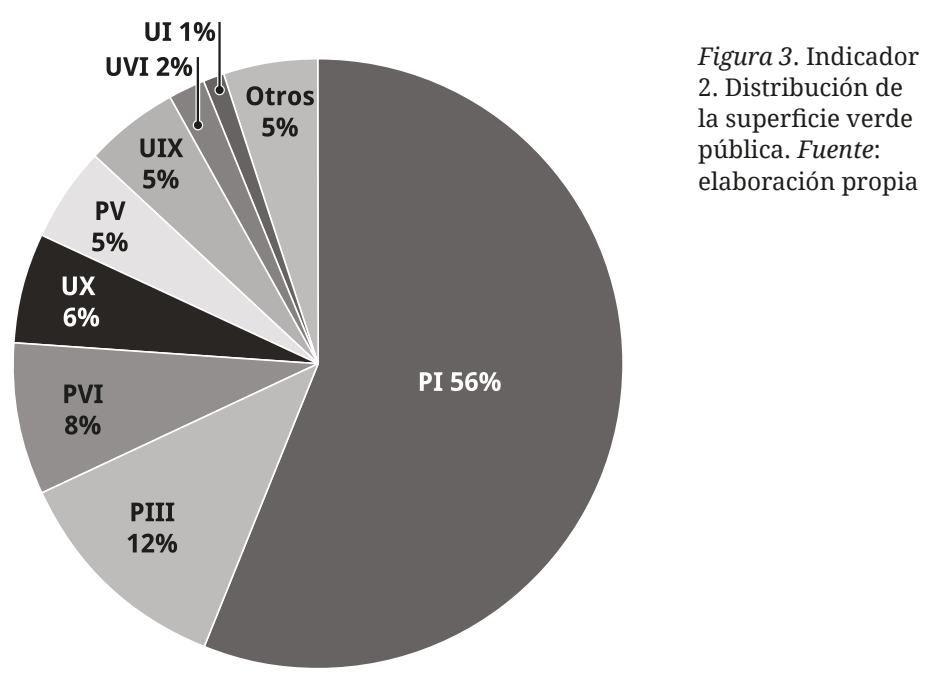

proviene casi exclusivamente de los espacios verdes públicos, en tanto el resto de la superficie exhibe valores en el rango que va de 0 a $25 \%$.

El periurbano, en cambio, presenta mayormente valores de entre 76 a $100 \%$, con algunos sectores de Batán y otros cercanos a Parque Camet que exhiben valores inferiores. No obstante, se advierte la existencia de algunos sectores con mayor superficie no absorbente por la presencia de cultivos bajo cubierta.

$\mathrm{Al}$ analizar las diferencias entre zonas, se observa que aquella con menor porcentaje de superficie no impermeabilizada es la UV (15,92 \%), la cual incluye un grupo de barrios con escasa superficie verde pública, que además tienen un tejido denso sin retiros de frente ni centros de manzana libre. En el extremo opuesto, las zonas con valores más altos en este indicador son la UX (51,95 \%) y la UVII (46,62 \%). Mientras en la primera el resultado se debe en parte a la presencia de espacios verdes públicos de gran tamaño, en el segundo caso, la mayor cantidad de superficie verde proviene de los jardines privados de las zonas residenciales, así como de la presencia de algunos sectores con menor nivel de consolidación, y por lo tanto con lotes sin construir y áreas de reserva urbana. 
Indicadores ambientales y gestión urbana.

Relaciones entre servicios ecosistémicos urbanos y sustentabilidad

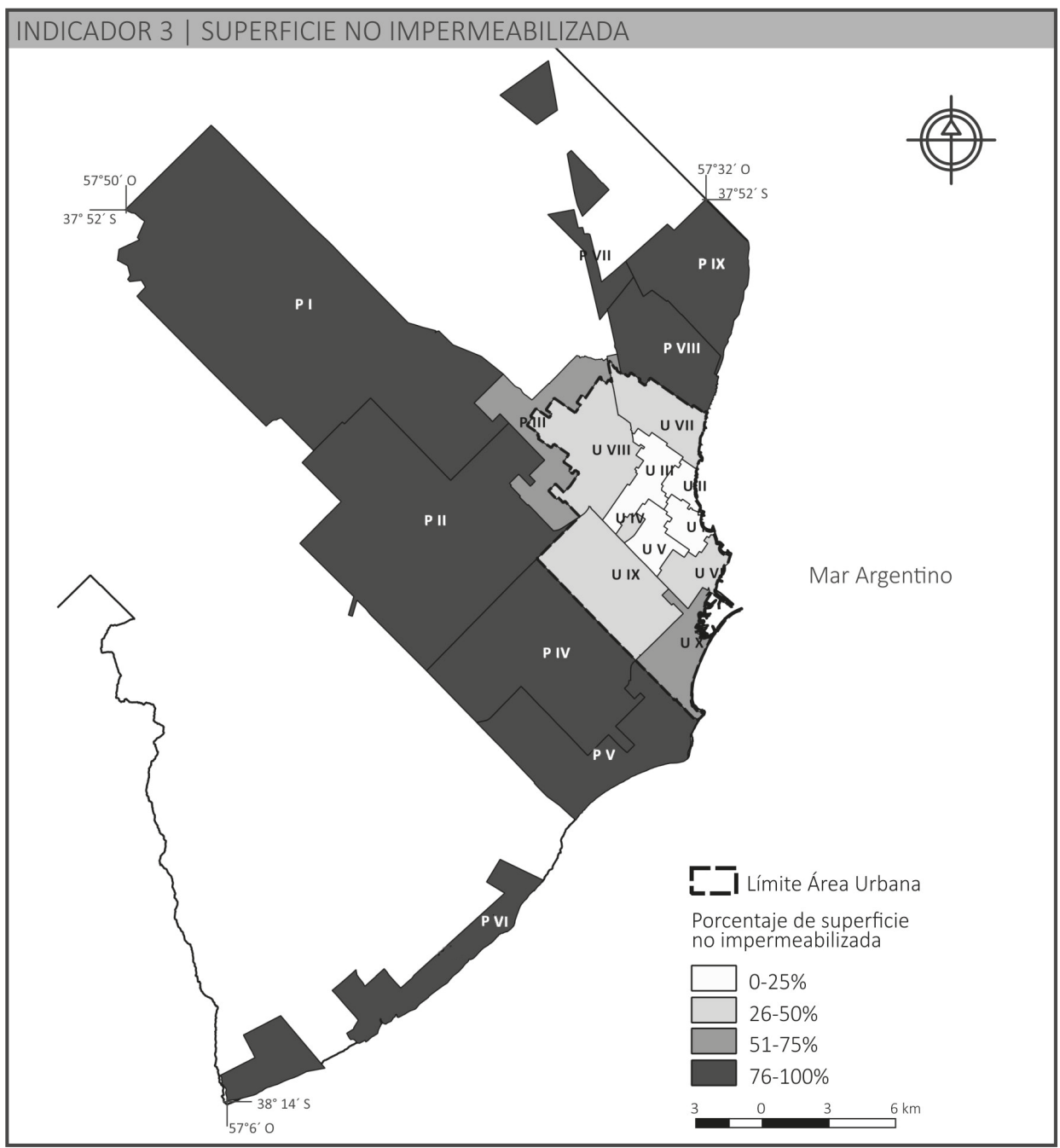

Figura 4. Indicador 3. Superficie no impermeabilizada. Fuente: elaboración propia 


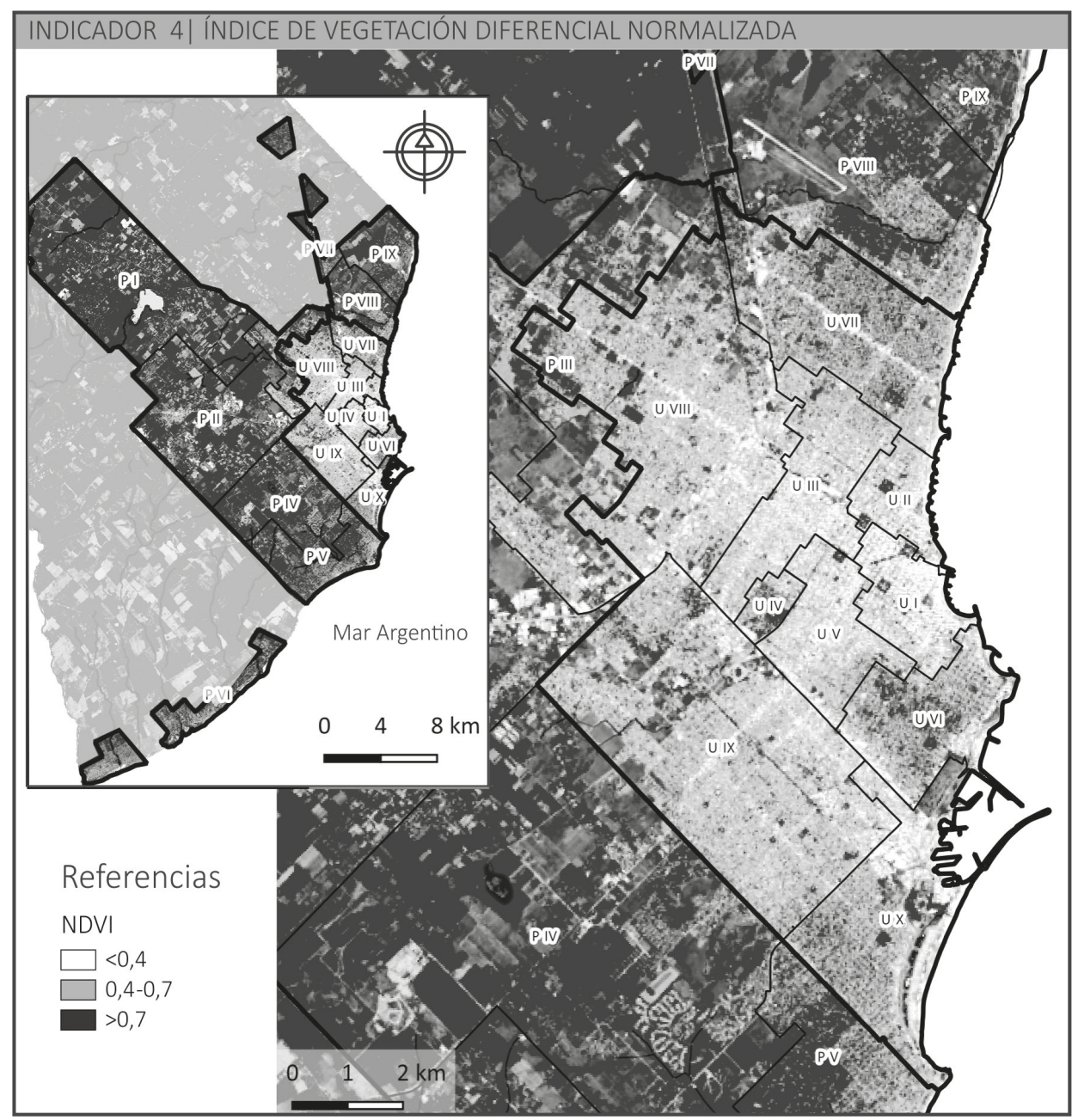

Figura 5. Indicador 4. NDVI. Fuente: elaboración propia 
Finalmente, los resultados del NDVI (figura 5) se relacionan con los distintos tipos de trazado, tejido y porcentaje de suelo impermeabilizado que caracterizan las zonas de análisis. Se advierte que, dentro del área urbana, los valores moderados a altos (> a 0,4) se encuentran en coincidencia con los espacios verdes públicos, grandes terrenos de reserva urbana y los barrios residenciales ubicados en las zonas UX, U V, UIV y UVII. No obstante, algunos escasos sectores exhiben valores de NDVI altos en áreas que presentan mayormente suelo construido, debido a la presencia de arbolado público. En cambio, en las zonas del periurbano los valores cercanos a cero corresponden principalmente a afloramientos rocosos y terrenos desnudos o sin vegetación, mientras los valores moderados a altos coinciden con zonas agrícolas o con barrios residenciales de baja densidad, algunos de ellos protegidos como reservas forestales. En estos casos, los valores de NDVI varían de acuerdo con el estado y densidad de la vegetación y los cultivos (Mujica,Karis y Ferraro , 2018).

\section{Conclusiones}

Los resultados de los indicadores evidenciaron la escasez de espacios verdes públicos en la mayor parte del área urbana de la ciudad de Mar del Plata, así como también en su periurbano. Asimismo, se observan diferencias respecto de la presencia de suelo cubierto de vegetación entre las zonas de análisis. De la comparación entre indicadores se observa que las zonas con mayor presencia de suelo cubierto con vegetación coinciden principalmente con barrios residenciales de baja densidad y alto porcentaje de suelo no impermeabilizado, que generalmente poseen escasa superficie verde pública (UIV, UV y UVII). En el extremo opuesto, el centro de la ciudad y las zonas exhiben valores bajos de vegetación, la cual proviene casi exclusivamente de los espacios verdes públicos identificados (UI y UII). Finalmente, se encontraron zonas con ausencia de vegetación y escasa superficie verde pública (UII y UVIII).

En este sentido, la ausencia de infraestructura ecológica podría tener implicancias en la posibilidad de contacto directo con la naturaleza por parte de la población del área de estudio, siendo esto un determinante en la generación de gran parte de los SEU culturales (Haines-Young y Potschin, 2018). Esta situación aleja el área de estudio de una condición de sustentabilidad, en cuya dimensión social se postula como uno de los criterios básicos la justicia distributiva (GUIMARÃEs, 2003). 
En cuanto a los alcances y limitaciones del presente trabajo, la metodología utilizada permitió establecer una línea de base para una serie de indicadores ambientales de la infraestructura ecológica proveedora de SEU culturales en la ciudad de Mar del Plata, a partir de los cuales se obtuvo una visión general respecto de la distribución de sus componentes dentro del área de estudio. Se trata de una primera aproximación a la temática, y se espera que, a partir de los resultados, sea posible profundizar en el análisis de los SEU culturales en el área de estudio e indagar respecto de las formas de valoración y percepción que los usuarios tienen sobre ellos. Asimismo, se espera que los datos obtenidos permitan también estudiar las relaciones existentes entre infraestructura ecológica, SEU culturales y otros tipos de servicios.

\section{Referencias bibliográficas}

AGENCIA DE ECOLOGÍA URBANA DE BARCELONA (2007). Capítulo V: El Libro Verde de Medio Ambiente Urbano en el Ámbito de la Gestión Urbana. En Parte I. El Libro Verde de Medio Ambiente Urbano (147-180). Barcelona: Ministerio de Medio Ambiente.

AGENCIA DE ECOLOGÍA URBANA DE BARCELONA (2010). Sistema de indicadores y condicionantes para ciudades grandes y medianas. España: Ministerio de Medio Ambiente y Medio Rural y Marino, Gobierno de España.

ANDERSSON, Erik; BARTHEL, Stephan y AHRNÉ, Karin (2007). Measuring social-ecological dynamics behind the generation of ecosystem services. Ecological Applications. 17(5), 1267-78.https://doi.org/10.1890/06-1116.1

BANCO INTERAMERICANO DE DESARROLLO (2016). Documento de Enfoque: Evaluación de la Iniciativa Ciudades Emergentes y Sostenibles. Recuperado de https:// publications.iadb.org/es/publicacion/17128/documento-de-enfoque-evaluacion-de-la-iniciativa-ciudades-emergentes-y

BARRERA DE LA, Francisco; REYES-PAECKE, Sonia y BANZHAF, Ellen (2016). Indicators for green spaces in contrasting urban settings. Ecological Indicators, 62, 212-219. https://doi.org/10.1016/j.ecolind.2015.10.027

BENEDICT, Mark y McMAHON, Edward (2002). Green Infrastructure: Smart Conservation for the 21st Century. Renewable Resources Journal, 20.

BRAAT, Leony y DE GROOT, Rudolf (2012). The ecosystem services agenda:bridging the worlds of natural science and economics, conservation and development, and public and private policy. Ecosystem Services. 1(1), 4-15. 
BREUSTE, Jürgen; HAASE, Dagmar y ELMQVIST, Thomas (2013). Urban Landscapes and Ecosystem Services. En Ecosystem Services in Agricultural and Urban Landscapes. https://doi.org/10.1002/9781118506271.ch6

BREUSTE, Jürgen; SCHNELLINGER, Johanna; QURESHI, Salman y FAGGI, Ana (2013). Urban Ecosystem services on the local level: Urban green spaces as providers. Ekologia Bratislava. 32(2), 290 - 304. https://doi.org/10.2478/ eko-2013-0026

CIVEIRA, Gabriela; LADO LIÑARES, Marcos; VIDAL VÁZQUEZ, Eva y PAZ GONZÁLEZ, Antonio (2018). Las áreas vegetadas en las ciudades y su aporte para mejorar la sustentabilidad ambiental. Perspectivas: Revista Científica de la Universidad de Belgrano, 1, 74-84.

COLES, Richard y BUSSEY, S. (2000). Urban forest landscapes in the UK - Progressing the social agenda. Landscape and Urban Planning. 52, 181-188.https://doi. org/10.1016/S0169-2046(00)00132-8

DICKINSON, Dawn y HOBBS, Richard (2017). Cultural ecosystem services: Characteristics, challenges and lessons for urban green space research. Ecosystem Services, 25, 179-94. https://doi.org/10.1016/j.ecoser.2017.04.014

EUROPEAN ENVIRONMENT AGENCY (2011). Green infrastructure and territorial cohesion: The concept of green infrastructure and its integration into policies using monitoring systems. EEA Tecnical Report. https://doi.org/10.2800/88266

GARAY, Alfredo (1999). Gestión ambiental de infraestructura y servicios urbanos. Texto correspondiente a materia de la Maestría en Gestión Ambiental del Desarrollo Urbano. Mar del Plata: Centro de Investigaciones Ambientales, FAUD-UNMdP.

GILABERT, M. A.; GONZÁLEZ-PIQUERAS, José y GARCÍA-HARO, Javier (1997). Acerca de los Índices de Vegetación. Revista de Teledetección, 8.

GÓMEZ-BAGGETHUN, Erik y BARTON, David (2013). Classifying and valuing ecosystem services for urban planning. Ecological Economics. 86, 235-245. https:// doi.org/10.1016/j.ecolecon.2012.08.019

GÓMEZ-BAGGETHUN, Erik; GREN, Åsa; BARTON, David; LANGEMEYER, Johannes; MCPHEARSON, Timon; O'FARRELL, Patrick... KREMER, Peleg (2013). Urban ecosystem services. En Urbanization, Biodiversity and Ecosystem Services: Challenges and Opportunities: A Global Assessment. Springer https://doi.org/10.1007/978-94-007-7088-1_11 
GROVE, Morgan (2009). Cities: Managing Densely Settled Social-Ecological Systems. En F. S. Chapin, G. P. Kofinas, \& C. Folke (Eds.), Principles of Ecosystem Stewardship (1.a ed., pp. 281-294). New York: Springer. https://doi.org/10.1007/978-0387-73033-2

GUIMARÃES, Roberto (2003). Tierra de sombras: desafios de la sustentabilidad y del desarrollo territorial y local ante la globalizacion. Polis. Revista Latinoamericana. 5.

HAINES-YOUNG, Roy y POTSCHIN, Marion (2018). Common International Classification of Ecosystem Services (CICES) V5.1 and Guidance on the Application of the Revised Structure. European Environment Agency.

KARIS, Clara y FERRARO, Rosana (2017). Identificación de la infraestructura verde y azul en la ciudad de Mar del Plata. Revista $I+A$. Facultad de Arquitectura Urbanismo y Diseño. Universidad Nacional de Mar del Plata, 20(19), 187-206.

KARIS, Clara (2017). Caracterización de los espacios verdes públicos en la ciudad de Mar del Plata, Argentina. Trabajo presentado en XXI Congreso ARQUISUR. Facultad de Arquitectura, Urbanismo y Diseño. Universidad Nacional de San Juan, Argentina.

KEELER, Bonnie; HAMEL, Perrine; MCPHEARSON, Timon; HAMANN, Maike; DONAHUE, Marie; MEZA PRADO, Kelly... WOOD, Spencer (2019). Social-ecological and technological factors moderate the value of urban nature. Nature Sustainability, 2(1), 29-38. https://doi.org/10.1038/ s41893-018-0202-1

LORAM, Alison; TRATALOS, Jamie; WARREN, Philip y GASTON, Kevin (2007). Urban domestic gardens $(\mathrm{X})$ : The extent \& structure of the resource in five major cities. Landscape Ecology. 22(4),601-615.https://doi.org/10.1007/s10980006-9051-9

LUCERO, Patricia; RIVIÉRE, Isabel; SAGUA, Maris; MIKKELSEN, Claudia; ARES, Sofía; AVENI, Silvina y SABUDA, Fernando (2011). Atlas Socio-territorial de Mar del Plata y el Partido de General Pueyrredón.

MARTÍN-LÓPEZ, Berta; GÓMEZ-BAGGETHUN, Erik y MONTES, Carlos (2009). Un marco conceptual para la gestión de las interacciones naturaleza- sociedad en un mundo cambiante. CUIDES. Cuaderno Interdisplinar de Desarrollo Sostenible, 3, 229-258. 
MEA (2003). Ecosistemas y Bienestar Humano: Marco para la Evaluación. Washington DC: Island Press.

MINISTERIO DE DESARROLLO URBANO BUENOS AIRES (2009). Atlas de Indicadores de Desarrollo Urbano de la Ciudad Autónoma de Buenos Aires. Ciudad Autónoma de Buenos Aires: Edición de la Subsecretaría de Planeamiento. Recuperado de https://www.buenosaires.gob.ar/planeamiento/publicaciones/atlas-de-indicadores-de-desarrollo-urbano

MUJICA, Camila; KARIS, Clara y FERRARO, Rosana (2018). Servicio ecosistémico urbano de regulación de temperatura en la ciudad de Mar del Plata durante la estación de verano. Póster presentado en las XXVIII Reunión Argentina de Ecología, Mar del Plata, 29 de octubre al 2 de noviembre.

MUNICIPALIDAD DE GENERAL PUEYRREDÓN (2017a). Código de Ordenamiento Territorial (COT). Recuperado de: http://appsvr.mardelplata.gob.ar/consultas/ appcontainer/appcontainer.asp?app=COT

MUNICIPALIDAD DE GENERAL PUEYRREDÓN (2017b). MGP Mapa digital. Recuperado de: https://gis.mardelplata.gob.ar/app_mapa_interactivo/planodigital.php?subrubro $=68$

QUIROGA MARTÍNEZ, Rayén (2009). Guía metodológica para desarrollar indicadores ambientales y de desarrollo sostenible en países de América Latina y el Caribe. CEPAL Serie Manuales.

REYES PÄCKE, Sonia y FIGUEROA ALDUNCE, Isabel (2010). Distribución, superficie y accesibilidad de las áreas verdes en Santiago de Chile. Eure. 36 (109), 89-110. https://doi.org/10.4067/S0250-71612010000300004

SANCHEZ RODRIGUEZ, Esperanza; TORRES CRESPO, Martín; PALACIOS CARMONA, Arturo; AGUILAR ALBA, Mónica; PINO SERRATO, Isabel y GRANADO RUIZ, Laura (2000). Comparación del NDVI con el PVI y el SAVI como indicadores para la asignación de modelos de combustible para la estimación del riesgo de incendios en Andalucía. En Tecnologías Geográficas para el Desarrollo Sostenible. Departamento de Geografía. Universidad de Alcalá: 164-174.Recuperado de: http://tig.age-geografia.es//docs/IX_2/Sanchez_Esperanza.PDF 
SANDSTRÖM, Ulf (2002). Green infrastructure planning in urban Sweden. Planning Practice and Research. 17(4), 373-385. https://doi.org/10.1080/02697450216356

TZOULAS, Konstantinos; KORPELA, Kalevi; VENN, Stephen; YLI-PELKONEN, Vesa; KA, Aleksandra; NIEMELA, Jari y JAMES, Philip (2007). Promoting ecosystem and human health in urban areas using Green Infrastructure : A literature review. Landscape and urban planning, 81, 167-178. https://doi. org/10.1016/j.landurbplan.2007.02.001

ZULAICA, Laura y FERRARO, Rosana (2013). Lineamientos para el ordenamiento del periurbano de la ciudad de Mar del Plata (Argentina), a partir de la definición de sistemas territoriales. Geografía em Questao, 06(1), 202-230. 